OPEN ACCESS

Edited by: John T. Fisher,

Queen's University, Canada

Reviewed by: François Marchal,

Centre Hospitalier Universitaire de Nancy, France

Norihiro Shinozuka

Chibaken Saiseikai Narashino

Hospital, Japan

*Correspondence: Walter A. Zin

walter_zin@hotmail.com

Specialty section: This article was submitted to Respiratory Physiology, a section of the journal

Frontiers in Physiology

Received: 19 December 2017

Accepted: 25 June 2018

Published: 13 July 2018

Citation:

Comuzzi L, de Abreu MB, Motta-Ribeiro GC, Okuro RT, Barboza T, Carvalho N, Lucangelo U,

Carvalho AR and Zin WA (2018)

Regional Lung Recruitability During

Pneumoperitoneum Depends on Chest Wall Elastance - A Mechanical and Computed Tomography Analysis in Rats. Front. Physiol. 9:920. doi: 10.3389/fphys.2018.00920

\section{Regional Lung Recruitability During Pneumoperitoneum Depends on Chest Wall Elastance - A Mechanical and Computed Tomography Analysis in Rats}

\author{
Lucia Comuzzi 1,2, Mariana B. de Abreu', Gabriel C. Motta-Ribeiro', Renata T. Okuro', \\ Thiago Barboza', Niedja Carvalho ${ }^{3}$, Umberto Lucangelo' ${ }^{2}$, Alysson R. Carvalho ${ }^{1,3}$ and \\ Walter A. Zin ${ }^{1 *}$
}

${ }^{1}$ Laboratory of Respiration Physiology, Carlos Chagas Filho Institute of Biophysics, Universidade Federal do Rio de Janeiro, Rio de Janeiro, Brazil, ${ }^{2}$ Department of Perioperative Medicine, Intensive Care and Emergency, Università degli Studi di Trieste, Trieste, Italy, ${ }^{3}$ Laboratory of Pulmonary Engineering, Alberto Luiz Coimbra Institute of Post-Graduation and Engineering Research, Universidade Federal do Rio de Janeiro, Rio de Janeiro, Brazil, ${ }^{4}$ National Center for Structural Biology and Bioimaging, Universidade Federal do Rio de Janeiro, Rio de Janeiro, Brazil

Background: Laparoscopic surgery with pneumoperitoneum increases respiratory system elastance due to the augmented intra-abdominal pressure. We aim to evaluate to which extent positive end-expiratory pressure (PEEP) is able to counteract abdominal hypertension preventing progressive lung collapse and how rib cage elastance influences PEEP effect.

Methods: Forty-four Wistar rats were mechanically ventilated and randomly assigned into three groups: control (CTRL), pneumoperitoneum (PPT) and pneumoperitoneum with restricted rib cage (PPT-RC). A pressure-volume (PV) curve followed by a recruitment maneuver and a decremental PEEP trial were performed in all groups. Thereafter, animals were ventilated using PEEP of 3 and $8 \mathrm{cmH}_{2} \mathrm{O}$ divided into two subgroups used to evaluate respiratory mechanics or computed tomography (CT) images. In 26 rats, we compared respiratory system elastance $\left(E_{r s}\right)$ at the two PEEP levels. In 18 animals, CT images were acquired to calculate total lung volume (TLV), total volume and air volume in six anatomically delimited regions of interest (three along the cephalo-caudal and three along the ventro-dorsal axes).

Results: PEEP of minimal $E_{r s}$ was similar in CTRL and PPT groups ( $3.8 \pm 0.45$ and $3.5 \pm 3.89 \mathrm{cmH}_{2} \mathrm{O}$, respectively) and differed from PPT-RC group (9.8 $\left.\pm 0.63 \mathrm{cmH}_{2} \mathrm{O}\right)$. Chest restriction determined a right- and downward shift of the PV curve, increased $\mathrm{E}_{\mathrm{rs}}$ and diminished TLV and lung aeration. Increasing PEEP augmented TLV in CTRL group $(11.8 \pm 1.3$ to $13.6 \pm 2 \mathrm{ml}, p<0.05)$, and relative air content in the apex of PPT group $(3.5 \pm 1.4$ to $4.6 \pm 1.4 \%$ TLV, $p<0.03)$ and in the middle zones in PPT-RC group ( $21.4 \pm 1.9$ to $25.3 \pm 2.1 \%$ TLV cephalo-caudally and $18.1 \pm 4.3$ to $22.0 \pm 3.3 \%$ TLV ventro-dorsally, $p<0.005)$. 


\begin{abstract}
Conclusion: Regional lung recruitment potential during pneumoperitoneum depends on rib cage elastance, reinforcing the concept of PEEP individualization according to the patient's condition.
\end{abstract}

Keywords: lung recruitability, PEEP, chest wall elastance, pneumoperitoneum, computed tomography scanning

\section{INTRODUCTION}

Laparoscopic surgery has been increasingly used as an alternative to open surgery for its well-known post-operative benefits. However, it is associated to intra-operative respiratory impairment (Valenza et al., 2010). Mechanical ventilation management is a challenge for the anesthetist in this scenario of intra-abdominal hypertension, because it is not clear how the modification of the ventilation parameters affects the different components of the respiratory system.

The respiratory system is composed of two elastic elements in series: the lung and the chest wall. The latter, in turn, is made up of two parallel components: the rib cage and the diaphragm, which is also part of the abdominal wall. Disregarding airflow resistances, the positive pressure applied to the respiratory system during mechanical ventilation distends all these elements. How the applied pressure is distributed within the respiratory system depends on the compliance of each single element (CortesPuentes et al., 2015) and its distribution within the lungs depends on the compliance of the lung zones, i.e., regional compliance (Mutoh et al., 1991; Lowhagen et al., 2010).

The production of a pneumoperitoneum with an intra-abdominal pressure $\left(\mathrm{P}_{\mathrm{ab}}\right)$ above the physiological is required to perform laparoscopic surgery. Andersson et al. (2005) showed by CT scans that pneumoperitoneum causes a cranial displacement of the diaphragm and stiffens it. Moreover, the dependent lung zones are more atelectatic and there is a reduction in pulmonary volumes (Andersson et al., 2005). As a result, respiratory system elastance $\left(\mathrm{E}_{\mathrm{rs}}\right)$ increases due to higher chest wall $\left(\mathrm{E}_{\mathrm{w}}\right)$ and lung $\left(\mathrm{E}_{\mathrm{L}}\right)$ elastances (Mutoh et al., 1991; Fahy et al., 1995; Moreira et al., 1997; Valenza et al., 2007a,b; MaracajáNeto et al., 2009; Futier et al., 2010; Formenti et al., 2012; Regli et al., 2012; Runck et al., 2012; Cinnella et al., 2013; CortesPuentes et al., 2013, 2015; Loring et al., 2014). PEEP triggers different outcomes in conditions of heterogeneous regional $\mathrm{E}_{\mathrm{L}}$ and $\mathrm{E}_{\mathrm{W}}$, e.g., intra-abdominal hypertension (Formenti et al., 2012; Regli et al., 2012; Runck et al., 2012; Cortes-Puentes et al., 2015). However, to our knowledge, the regional PEEP distribution within the lungs during pneumoperitoneum has not been so far described.

We aimed to evaluate the recruitment potential of two different PEEP levels in rats with modified chest wall compliance by pneumoperitoneum alone and pneumoperitoneum plus restricted chest. The assessment was done globally, by means

Abbreviations: CT scan, computed tomography scan; $\mathrm{E}_{\mathrm{L}}$, lung elastance; $\mathrm{E}_{\mathrm{rs}}$, respiratory system elastance; $\mathrm{E}_{\mathrm{w}}$, chest wall elastance; $\mathrm{FiO}_{2}$, inspired oxygen fraction; I:E, inspiratory to expiratory time ratio; $\mathrm{PaO}_{2} / \mathrm{FiO}_{2}$, arterial oxygen partial pressure to inspired oxygen fraction ratio; $\mathrm{P}_{\mathrm{ab}}$, intra-abdominal pressure; $\mathrm{P}_{\mathrm{aw}}$, airway pressure; PEEP, positive end-expiratory pressure; PEEPmin-Ers, PEEP at minimal elastance; PV curve, pressure-volume curve; ROI, region of interest; RR, respiratory rate; TLC, total lung capacity; TLV, total lung volume; $\mathrm{V}_{\mathrm{T}}$, tidal volume. of respiratory mechanics, and regionally, by quantitative CT imaging. We hypothesized that the more restricted is the chest, the larger is the PEEP recruitment potential.

\section{MATERIALS AND METHODS}

\section{Animals and Study Groups}

Forty-eight male Wistar rats (330-430 g) were used. All received human care in compliance with the National Institutes of Health Guide for the Care and Use of Laboratory Animals (NIH Publications No. 8023, revised 1978), and National Council for Controlling Animal Experimentation, Ministry of Science, Technology and Innovation (CONCEA/MCTI), Brazil. The Ethics Committee on the Use of Animals, Health Sciences Centre, Federal University of Rio de Janeiro approved the experimental protocol (103/2013).

Rats were housed in groups of five animals per cage (Makrolon polycarbonate Type IV) and food and water were provided ad libitum. They were randomly divided using sealed envelopes into three groups: control (CTRL), pneumoperitoneum (PPT) and pneumoperitoneum with restricted chest (PPT-RC). Twelve animals were allocated in CTRL group and 16 in each of the other two groups. Each group was further divided into two subgroups used to evaluate respiratory mechanics or CT images. Six animals from each group underwent whole lung CT scans in a preclinical micro-CT/PET/SPECT scanner (Tri-Modality FLEX Triumph Pre-Clinical Imaging System, Gamma Medica-Ideas, Northridge, CA, United States). The remaining 26 animals were used for measurement of global respiratory system mechanics (6 in CTRL and 10 in PPT and PPT-RC groups).

Sample size was determined to reach a power of $80 \%$ and a significance level of $1.67 \%$ (5\% corrected for the three-group comparison), with an effect size of 0.9 . Only 12 rats were allocated to CTRL group because it was expected to be more homogenous.

\section{Experimental Protocol}

Animals were anesthetized with isoflurane $2.5 \%$ vol with a calibrated vaporizer. A $24-\mathrm{G}$ catheter was indwelled into a tail vein and a $20-G$ catheter into the right carotid artery. Then, the rats were tracheotomized, paralyzed with pancuronium bromide $(0.2 \mathrm{mg} / \mathrm{kg} \mathrm{IV})$ and mechanically ventilated (Inspira ASV, Harvard Apparatus, Holliston, MA, United States) in volume-controlled ventilation mode with tidal volume $\left(\mathrm{V}_{\mathrm{T}}\right)$ of $8 \mathrm{ml} / \mathrm{kg}$, respiratory rate (RR) of 70 breaths/min, inspiratory to expiratory time ratio (I:E) of 1:2, PEEP of $5 \mathrm{cmH}_{2} \mathrm{O}$ and inspired oxygen fraction ratio $\left(\mathrm{FiO}_{2}\right)$ of 0.5 .

Afterward, $1 \mathrm{ml}$ of a colloid solution (6\% hydroxyethyl starch 130/0.42, Centralvet, Vinhedo, SP, Brazil) was administered intravenously and a recruitment maneuver was performed by 
setting PEEP at $15 \mathrm{cmH}_{2} \mathrm{O}$ for five consecutive breathing cycles. After the procedure, PEEP was set at $5 \mathrm{cmH}_{2} \mathrm{O}$, and, after $5 \mathrm{~min}$, arterial blood was collected for gas analysis (T0, i-STAT with EG4 ${ }^{+}$Cartridge, Abbott, Chicago, IL, United States). If arterial oxygen partial pressure to inspired oxygen fraction $\left(\mathrm{PaO}_{2} / \mathrm{FiO}_{2}\right)$ was $\geq 400$, the experimental protocol started. If not, the maneuver was repeated with a PEEP of $20 \mathrm{cmH}_{2} \mathrm{O}$ and if $\mathrm{PaO}_{2} / \mathrm{FiO}_{2}$ was $\geq 400$ the experimental protocol began. If after the recruitment maneuvers $\mathrm{PaO}_{2} / \mathrm{FiO}_{2}$ remained $<400$, the animal was cast aside.

Before pneumoperitoneum production, $2 \mathrm{ml}$ of colloid were administered intravenously in the PPT and PPT-RC groups. Then, a 16-G catheter was inserted through and fixed to the abdominal wall, and nitrogen was used for abdominal insufflation. We used an inert gas because during CT scan it was not possible to maintain the animal connected to the insufflation system and adjust gas pressure. The target $\mathrm{P}_{\mathrm{ab}}$ was 16-20 $\mathrm{cmH}_{2} \mathrm{O}$. In PPT-RC group, chest restriction was performed with a sphygmomanometer (number 4) wrapped around the thorax of the animal and inflated until a pressure of $12-15 \mathrm{cmH}_{2} \mathrm{O}$ was reached. Two pressure transducers (UT-PDP-50, SCIREQ, Montreal, QC, Canada), connected to a computer, controlled $\mathrm{P}_{\mathrm{ab}}$ and the insufflation pressure of the sphygmomanometer.

Thereafter, the inspiratory limb of pressure-volume (PV) curves was obtained with a low-flow inflation by adjusting PEEP to $0 \mathrm{cmH}_{2} \mathrm{O}, \mathrm{RR}$ to 5 breaths/min, $\mathrm{I}$ : $\mathrm{E}$ ratio to $4: 1$ and $\mathrm{V}_{\mathrm{T}}$ to $30 \mathrm{~mL} / \mathrm{kg}$. The resulting inspiratory flow was $<200 \mathrm{ml} / \mathrm{min}$. Four PV curves were performed and that presenting a stable peak airway pressure (circa $35 \mathrm{cmH}_{2} \mathrm{O}$ ) was considered for further analysis (Carvalho et al., 2013).

Right after gathering the PV curve, a recruitment maneuver with the formerly successful PEEP (15 or $20 \mathrm{cmH}_{2} \mathrm{O}$ ) and a decremental PEEP trial were performed. For the latter, PEEP was set at $10 \mathrm{cmH}_{2} \mathrm{O}$ and decreased to $1 \mathrm{cmH}_{2} \mathrm{O}$ in unitary steps lasting $30 \mathrm{~s}$ each (Carvalho et al., 2013).

All animals were then ventilated using two different PEEPs, 3 and $8 \mathrm{cmH}_{2} \mathrm{O}$. Firstly, ventilation was performed with PEEP of either 3 or $8 \mathrm{cmH}_{2} \mathrm{O}$ for $10 \mathrm{~min}$, and, then, they were ventilated for another $10 \mathrm{~min}$ with the other PEEP. The sequence was determined in a random fashion using sealed envelopes. Before applying each PEEP, all animals were maintained at PEEP $=1$ $\mathrm{cmH}_{2} \mathrm{O}$ for $5 \mathrm{~min}$, to maintain volume history. At the end of the experiment, euthanasia was performed by sectioning the inferior vena cava and abdominal aorta under deep anesthesia (isoflurane $5 \% \mathrm{vol})$.

\section{Data Acquisition and Processing}

Airway pressure $\left(\mathrm{P}_{\mathrm{aw}}\right)$ and flow $\left(\mathrm{V}^{\prime}\right)$ were continuously measured using a heated pneumotachograph (8430B, Hans Rudolph, Shawnee, KS, United States) connected between the endotracheal tube and the Y-piece of the ventilatory circuit. The pneumotachograph was connected to two differential pressures transducers, one for $\mathrm{P}_{\mathrm{aw}}$ (UT-PDP-50, SCIREQ, Montreal, QC, Canada) and another for $\mathrm{V}^{\prime}$ (UT-PDP-02, SCIREQ, Montreal, QC, Canada) measurements. $\mathrm{P}_{\mathrm{aw}}$ and $\mathrm{V}^{\prime}$ signals were low-pass filtered at $30 \mathrm{~Hz}$, digitized at $1000 \mathrm{~Hz}$ using a 14-bit analog-to-digital converter (NI-6009, National Instruments,
Austin, TX, United States) and recorded with a built-purpose routine written in LabVIEW (National Instruments, Austin, TX, United States). All transducers were calibrated before the experiments.

$\mathrm{V}_{\mathrm{T}}$ was calculated by numerical integration of $\mathrm{V}^{\prime}$ and $\mathrm{P}_{\mathrm{aw}}$ was fitted on a breath-by-breath basis to a linear single-compartment model (Eq. 1) for the estimation of respiratory system elastance $\left(\mathrm{E}_{\mathrm{rs}}\right)$ :

$$
\operatorname{Paw}(t)=\operatorname{Ers} \cdot V(t)+R r s \cdot V^{\prime}(t)+E E P
$$

where $R_{r s}$ is the respiratory system resistance, EEP is the end-expiratory pressure at zero $\mathrm{V}^{\prime}$ and volume $(\mathrm{V})$ and $\mathrm{t}$ is time.

The mechanical parameters were estimated considering the mean value from the last 20 cycles at the end of each PEEP step during PEEP titration and 40 cycles at each PEEP used during the ventilation protocol (PEEP 1, 3, and $8 \mathrm{cmH}_{2} \mathrm{O}$ ). The PEEP at minimal $\mathrm{E}_{\mathrm{rs}}$ (PEEPmin-Ers) was calculated as the minimum of a third degree polynomial fitted to $\mathrm{E}_{\mathrm{rs}}$ versus PEEP curve during the titration maneuver. Additionally, we compared $\mathrm{E}_{\mathrm{rs}}$ using $\mathrm{PEEP}=3$ vs. $8 \mathrm{cmH}_{2} \mathrm{O}$ in each group and among groups.

\section{Computed Tomography Scan Acquisition and Processing}

Computed tomography scan was performed with a small animal micro-PET/SPECT/CT scan (Tri-Modality FLEX Triumph Pre-Clinical Imaging System, Gamma Medica-Ideas, Northridge, CA, United States). The acquisition protocol was based on 5 -mm-thick axial slices, $1.5 \mathrm{x}$ collimation, FOV 78.92, Binning $2 \times 2,3$ frames of 1024 slices, $75 \mathrm{kVp}$ and $135 \mu$ As. The procedure for each CT scan lasted about $10 \mathrm{~min}$.

In all images, lung parenchyma was segmented semi-automatically using a threshold and growing region algorithm with multiple seeds in Osirix software (Pixmeo, Geneva, Switzerland). Data were then exported as DICOM files for images, and HDR+IMG for regions of interest (ROI), to be later processed in MATLAB (MathWorks, Natick, MA, United States) using a custom code.

We divided the lungs in three ROI along the cranio-caudal axis (apex, middle, and base) and three ROI along the ventro-dorsal axis (ventral, middle, and dorsal). The division obeyed visually identified anatomical landmarks: the carina and the heart apex for the cranio-caudal axis, and the top of the heart (considering the heart zone closest to the sternum in a lateral view) and the top of the hilum (approximately the entrance of the airways into the lungs) for the ventro-dorsal axis. We calculated and compared TLV using PEEP $=3$ vs. $8 \mathrm{cmH}_{2} \mathrm{O}$ in each group and also among the groups. Additionally, we calculated and compared the total volume and the air volume of each ROI using $\mathrm{PEEP}=3$ vs. $8 \mathrm{cmH}_{2} \mathrm{O}$ in each group. To account for differences in lung dimensions, the volumes of the ROIs were normalized by TLV.

Finally, we processed CT images to describe the lung density distribution, expressed in Hounsfield units, in the three groups using $\mathrm{PEEP}=3$ and $8 \mathrm{cmH}_{2} \mathrm{O}$. The integral of the curves corresponds to TLV. 


\section{Statistical Analysis}

Intra-group comparisons were performed with paired $t$-test and inter-group comparisons with one-way ANOVA. Multiple comparisons were corrected with the Bonferroni-Holm's method. All analysis were performed in MATLAB (MathWorks, Natick, MA, United States) considering $\alpha=5 \%$.

\section{RESULTS}

Three animals of PPT-RC group were excluded because baseline $\mathrm{PaO}_{2} / \mathrm{FiO}_{2}$ was $\leq 400 \mathrm{mmHg}$ after the second lung recruitment maneuver and one rat of PPT group died during micro-CT scanning. These rats were replaced by animals from the same batch. We were unable to properly reconstruct the CT image in one CTRL animal, therefore only images of five rats were analyzed in that group.

\section{Respiratory Mechanics}

Normalized inspiratory PV curves of all animals are shown in Figure 1. CTRL group (Figure 1A) showed sigmoidal PV curves with discreet lower and evident upper inflection points, suggesting the predominance of overdistension in relation to recruitment. In the PPT group, linear PV curves were observed (Figure 1B), whereas in the PPT-RC group the upward volume/pressure concavity suggests a progressive recruitment throughout inflation (Figure 1C). TLCs were $10.9 \pm 1.0$, $9.1 \pm 1.5$, and $7.3 \pm 2.2 \mathrm{ml}$ in CTRL, PPT, and PPT-RC groups, respectively.

Figure 2 presents $E_{r s}$ versus PEEP in each group during the decremental PEEP trial. In CTRL group (Figure 2A) $\mathrm{E}_{\mathrm{rs}}$ progressively fell with decreasing PEEP up to a PEEP of $3.8 \pm 0.4$ $\mathrm{cmH}_{2} \mathrm{O}$. Then, a slight increase in $\mathrm{E}_{\mathrm{rs}}$ was observed, which resulted in a J-shaped profile. In PPT group (Figure 2B) $\mathrm{E}_{\mathrm{rs}}$ was almost constant throughout the entire PEEP-trial (minimal $\mathrm{E}_{\mathrm{rs}}$ at PEEP of $3.5 \pm 3.89 \mathrm{cmH}_{2} \mathrm{O}$ ), whereas in the PPT-RC group (Figure 2C) $\mathrm{E}_{\mathrm{rs}}$ increased while PEEP decreased reaching a minimal close to the highest tested PEEP at $9.8 \pm 0.6 \mathrm{cmH}_{2} \mathrm{O}$.

During the ventilation protocol, $\mathrm{E}_{\mathrm{rs}}$ increased progressively with increasing chest restriction, independently of the PEEP applied (Figure 3). $\mathrm{E}_{\mathrm{rs}}$ was higher in PEEP $=8 \mathrm{cmH}_{2} \mathrm{O}$ than in PEEP $=3 \mathrm{cmH}_{2} \mathrm{O}$ in groups CTRL and PPT, and smaller in PPT-RC group. $\mathrm{E}_{\mathrm{rs}}$ was smaller in $\mathrm{PEEP}=3 \mathrm{cmH}_{2} \mathrm{O}$ than in $\mathrm{PEEP}=1 \mathrm{cmH}_{2} \mathrm{O}$ in CTRL group (without clinical relevance) and higher in the other two groups. Elastance was higher in PEEP $=8$ $\mathrm{cmH}_{2} \mathrm{O}$ than in PEEP $=1 \mathrm{cmH}_{2} \mathrm{O}$ in CTRL and PPT groups but diminished in PPT-RC group (Figure 3 ). In all groups the two measurements under PEEP $=1 \mathrm{cmH}_{2} \mathrm{O}$ did not differ.

\section{CT Scan Images}

Figure 4 depicts examples of lung surface reconstructions obtained by processing CT scan images and the corresponding TLV for each group and PEEP.

Figure 5 represents histograms showing mean lung density, which is correlated with lung aeration (Gattinoni et al., 1987), expressed in Hounsfield units, in the three groups at PEEPs $=3$ and $8 \mathrm{cmH}_{2} \mathrm{O}$. Aeration has a unimodal distribution in all cases. With increasing chest restriction, a rightward shift of the curves, i.e., a decreasing aeration can be seen.

Total lung volume decreased progressively with increasing chest wall restriction, independently of the PEEP used $(p<0.001$ in all comparisons). Increasing PEEP augmented TLV in CTRL group $(11.8 \pm 1.3$ to $13.6 \pm 2 \mathrm{ml}, p=0.036)$, while in the other groups TLV did not change (PPT group: $7.3 \pm 0.7$ and $8.1 \pm 1.4 \mathrm{ml}$, PPT-RC group: $6.1 \pm 0.7$ and $6.2 \pm 0.8 \mathrm{ml}, p=0.09$ and 0.66 , respectively). On the average, the percentage variations of TLV from PEEP 3 to $8 \mathrm{cmH}_{2} \mathrm{O}$ were +15.2 , +11 , and $+1.6 \%$ in CTRL, PPT and PPT-RC groups, respectively.

In addition, we analyzed the volume and the density of six ROIs, considering the cephalo-caudal (apex, middle, and base) and the ventro-dorsal (ventral, middle, and dorsal) axes. Figure 6 shows the fractional contribution of total volume of each ROI to TLV (Figures 6A,B) and the fractional contribution of the air content of each ROI to TLV (Figures 6C,D), at both PEEP levels in each group. With increasing PEEP, the middle cephalo-caudal zone presented a smaller contribution to TLV in CTRL group ( $48.2 \pm 5$ to $44.4 \pm 4.8 \%, p=0.013$ ); in PPT group, the apex air content exhibited a higher contribution to TLV with PEEP $=8$ $\mathrm{cmH}_{2} \mathrm{O}(3.5 \pm 1.4$ to $4.6 \pm 1.4 \%, p=0.027)$; in PPT-RC group, the base presented a reduced contribution $(37.5 \pm 6.1$ to
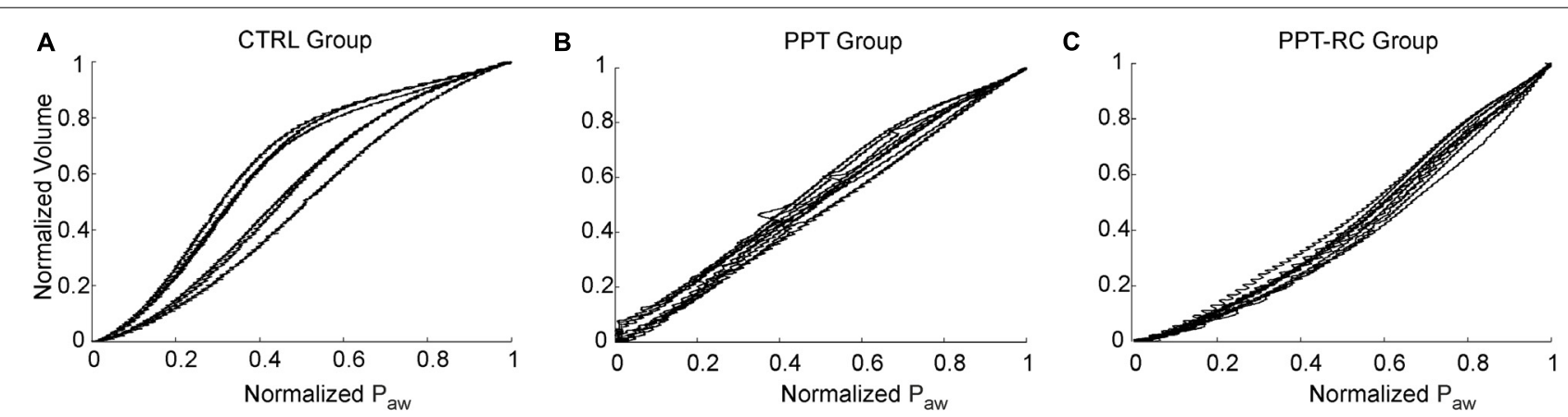

FIGURE 1 | Inspiratory limb of pressure versus volume (PV) curves in the three experimental groups (A-C) obtained with the low flow inflation. Each line represents one animal. Airway pressure $\left(\mathrm{P}_{\mathrm{aw}}\right)$ and volume are normalized considering the maximum and minimum value of each animal. CTRL, control group; PPT, animals with pneumoperitoneum; PPT-RC, animals with pneumoperitoneum and rib cage restriction. 

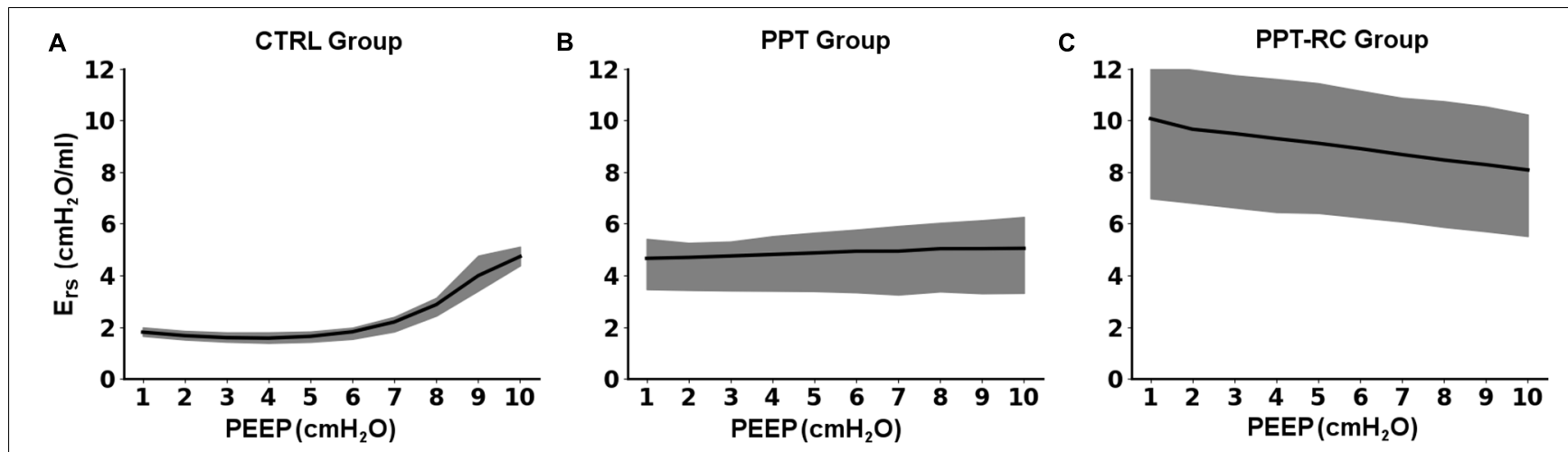

FIGURE 2 | Changes in respiratory system elastance $\left(E_{r s}\right)$ as a function of PEEP during the decremental PEEP trial in the three experimental groups (A-C). Black line and gray band represent mean values and standard deviations, respectively. CTRL, control group; PPT, animals with pneumoperitoneum; PPT-RC, animals with pneumoperitoneum and rib cage restriction.

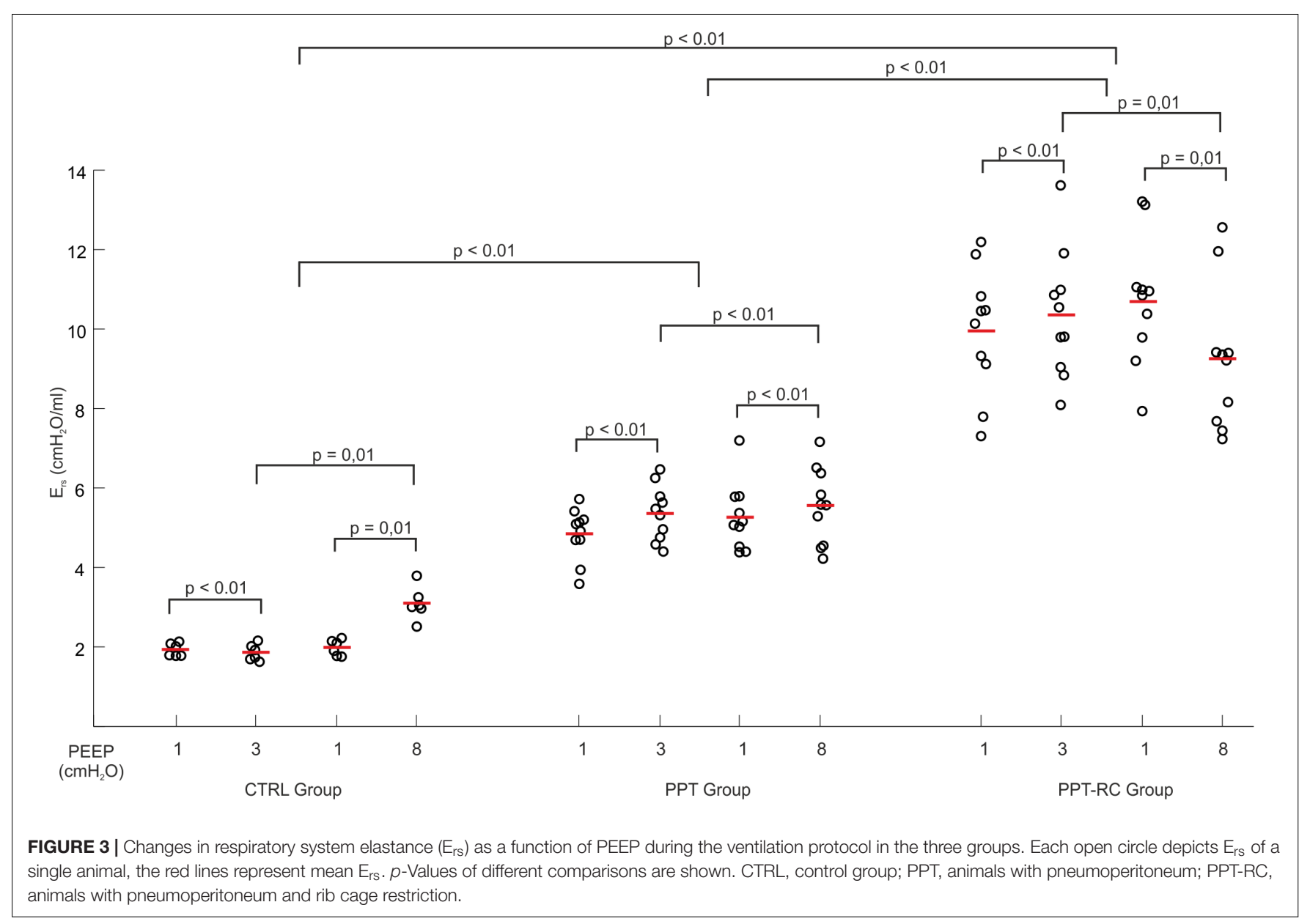

$35.9 \pm 5.9 \%, p=0.023)$ and the middle ventro-dorsal zone an increased contribution to TLV with PEEP $=8 \mathrm{cmH}_{2} \mathrm{O}(45 \pm 7.2$ to $48.9 \pm 6.8 \%, p=0.023)$. Moreover, in PPT-RC group the middle ventro-dorsal $(18.1 \pm 4.3$ to $22 \pm 3.3 \%, p=0.003)$ and cephalo-caudal zones $(21.4 \pm 1.9$ to $25.3 \pm 2.1 \%, p=0.003)$ increased their air volume relative to TLV under PEEP $=8$ $\mathrm{cmH}_{2} \mathrm{O}$.

\section{DISCUSSION}

The effects of pneumoperitoneum and PEEP on respiratory mechanics and aeration are well-known (Fahy et al., 1995; Moreira et al., 1997; Andersson et al., 2005; Valenza et al., 2007a,b; Maracajá-Neto et al., 2009; Futier et al., 2010; Formenti et al., 2012; Regli et al., 2012; Runck et al., 2012; Cinnella et al., 2013; 

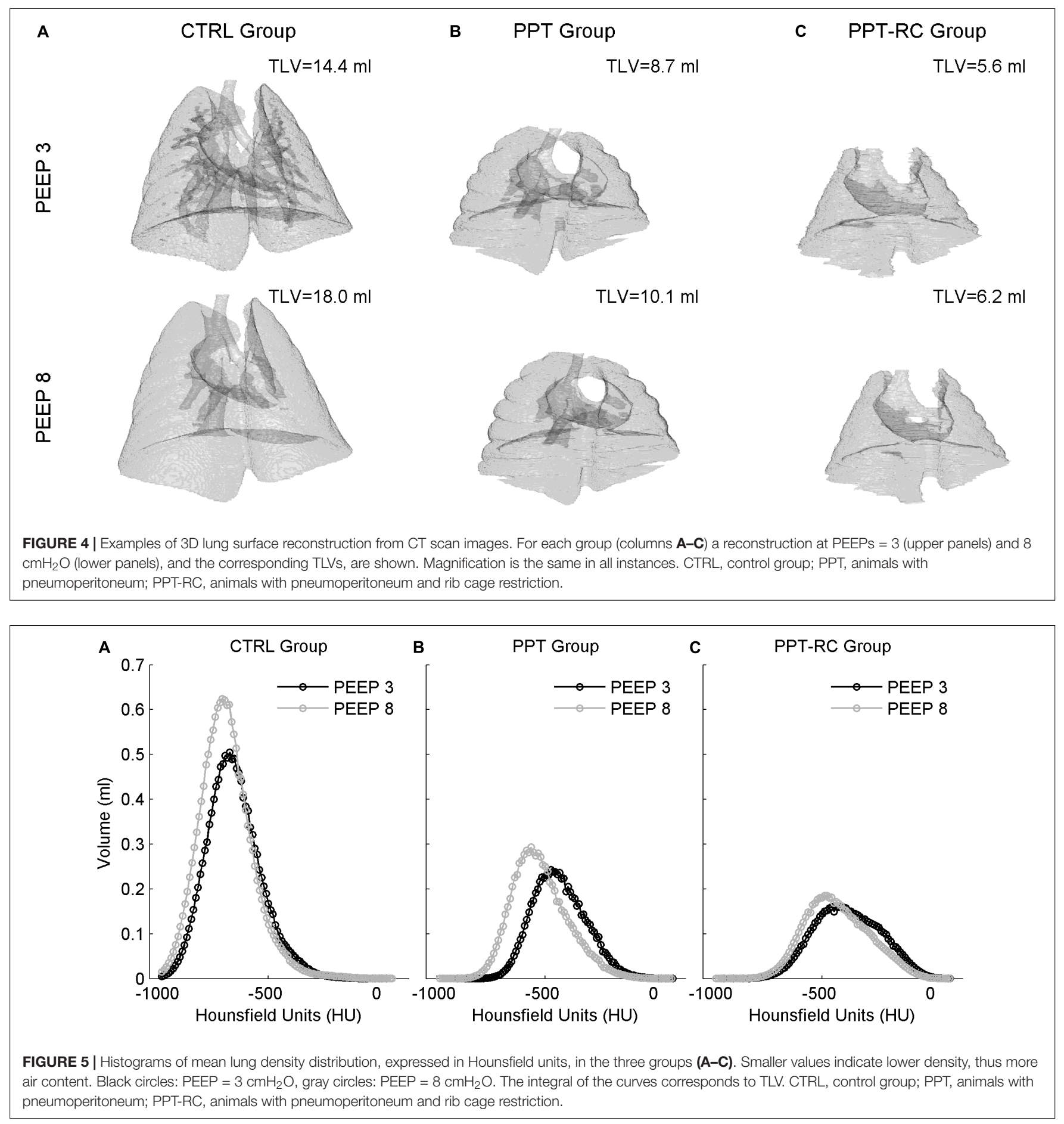

Cortes-Puentes et al., 2013; Loring et al., 2014; Cortes-Puentes et al., 2015). However, there is no previous investigation about the consequences of different PEEP levels in the presence of pneumoperitoneum and variable $\mathrm{E}_{\mathrm{w}}$ on respiratory mechanics and lung regional aeration.

The restriction of the ribcage was included in our study for three reasons: firstly, rats have naturally a more compliant chest wall than humans (Loring et al., 2010); secondly, we wanted to homogenize ribcage elastance among rats; thirdly, we believed that further increasing $\mathrm{E}_{\mathrm{w}}$ would improve the effect of PEEP on lung recruitability.

Our main findings were: (1) the more restricted is the chest wall, the higher is $\mathrm{E}_{\mathrm{rs}}$ and the lower is TLV, independently of PEEP; (2) PEEP of minimal $\mathrm{E}_{\mathrm{rs}}$ is higher in the face of ribcage restriction; (3) accordingly, lung recruitability during intra-abdominal hypertension induced 

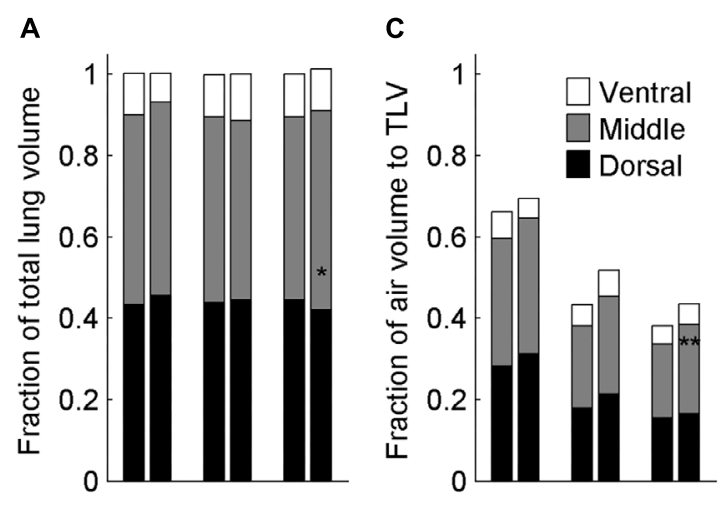

B
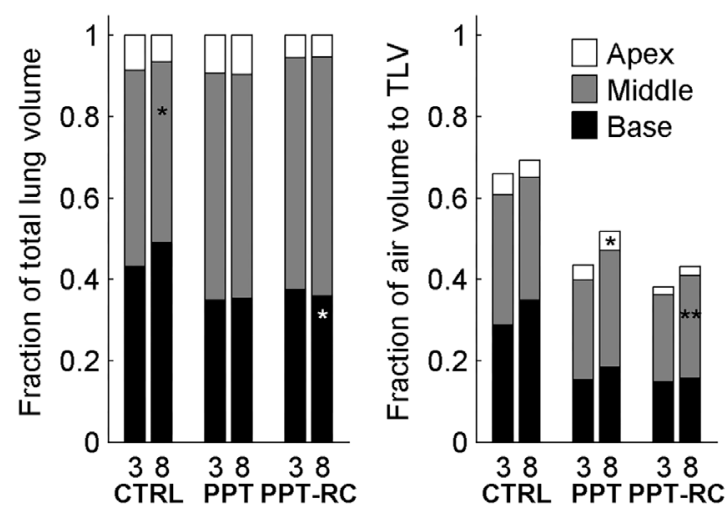

FIGURE 6 | Fractional contribution of the volume of the regions of interest ( $\mathrm{ROI}$, three along the ventro-dorsal and three along the cephalocaudal axes) to TLV in the three groups at PEEP $=3$ and $8 \mathrm{cmH}_{2} \mathrm{O}(\mathbf{A}, \mathbf{B})$ and fractional contribution of the ROl's air volume to TLV in the three groups at PEEP $=3$ and $8 \mathrm{cmH}_{2} \mathrm{O}(\mathbf{C , D})$. Asterisks indicate statistically significant intra-group differences between PEEP $=3$ and $8 \mathrm{cmH}_{2} \mathrm{O}$ in each zone $\left(^{*} p<0.05\right.$, $\left.{ }^{* *} p<0.01\right)$. CTRL, control group; PPT, animals with pneumoperitoneum; PPT-RC, animals with pneumoperitoneum and rib cage restriction.

by pneumoperitoneum depends on the elastance of the ribcage.

\section{PV Curves}

The PV curves of CTRL group showed the classical shape of rats' PV curves, with a lower and a more distinct upper corner points (Martin-Lefèvre et al., 2001; Loring et al., 2010).

Restricting the entire chest wall, i.e., rib cage and abdominal compartments (PPT-RC group), led to a decrease in the slope of the curve, the disappearance of the upper corner, and a decreased final volume, in accordance with previous findings in rats with restricted ribcage (Loring et al., 2010).

Pneumoperitoneum alone (PPT group) produced similar and less important curve alterations, as previously reported (Mutoh et al., 1991; Valenza et al., 2007a,b; Runck et al., 2012; Loring et al., 2014). Moreover, the lower corner disappeared. This may be due to the high compliance of the rib cage that is expanded by PEEP, thus consuming pressure that would be otherwise available for lung recruitment. This supports our hypothesis about the role of the rib cage in determining the recruitment potential of PEEP in the presence of pneumoperitoneum.

\section{PEEP Titration Curves and Ventilation Protocol: PEEP = 3 Versus $8 \mathrm{cmH}_{2} \mathrm{O}$}

We compared two PEEP levels. The lower level (PEEP $=3$ $\mathrm{cmH}_{2} \mathrm{O}$ ) was chosen because it is close to the mean value of PEEPmin-Ers in rats (Carvalho et al., 2013; Camilo et al., 2014) and the higher level $\left(\mathrm{PEEP}=8 \mathrm{cmH}_{2} \mathrm{O}\right)$ was chosen aiming at counteracting the increased $\mathrm{P}_{\mathrm{ab}}$, since $\mathrm{E}_{\mathrm{rs}}$ starts to increase at PEEP levels close to $8 \mathrm{cmH}_{2} \mathrm{O}$ (Figure 1A) in the CTRL group. It could be suggested that at this level overdistension might occur, as previously reported (Formenti et al., 2012).

In CTRL group, we obtained the expected $\mathrm{E}_{\mathrm{rs}}$ vs. PEEP titration curve profile (Figure 2A), with a flat region of PEEP of minimal $\mathrm{E}_{\mathrm{rs}}$, which corresponds to the best balance between tidal recruitment and hyperinflation, higher levels of PEEP leading to overdistention and lower ones to excessive derecruitment (Carvalho et al., 2006, 2008). During the ventilation protocol, as expected, $\mathrm{E}_{\mathrm{rs}}$ was lower with $\mathrm{PEEP}=3 \mathrm{cmH}_{2} \mathrm{O}$ than with $\mathrm{PEEP}=8 \mathrm{cmH}_{2} \mathrm{O}$ (Figure 3 ), probably because there is no chest wall restriction associated with some degree of lung overdistension (Regli et al., 2012; Runck et al., 2012). Possibly the upper low compliance part of the PV curve was reached.

In PPT group, we obtained almost constant $\mathrm{E}_{\mathrm{rs}}$ as a function of PEEP (Figure 2B). During the ventilation protocol, $\mathrm{E}_{\mathrm{rs}}$ was always higher than in CTRL rats, suggesting that lung volume was reduced due to abdominal hypertension (Figures 3, 4B), likely leading to alveolar derecruitment. Probably, the balance between $\mathrm{P}_{\mathrm{ab}}$ and PEEP was not sufficient to recruit the lung (Regli et al., 2012; Runck et al., 2012; Cortes-Puentes et al., 2013). Additionally, Figure 1B shows that elastance was constant throughout the total PV curve.

In PPT-RC group, $\mathrm{E}_{\mathrm{rs}}$ was always higher than in the other groups independently of PEEP level. Possibly, the increased $\mathrm{P}_{\mathrm{ab}}$ associated with chest wall restriction promoted an important fall in lung volume (Figure 4C) that augmented $\mathrm{E}_{\mathrm{rs}}$ even further. PEEP titration showed a decreasing $\mathrm{E}_{\mathrm{rs}}$ with increasing pressures (Figure 2C), and PEEPmin-Ers corresponded to the highest PEEP step. This suggests that the range of PEEP tested was too narrow, and that the real PEEPmin-Ers is likely to be higher (Loring et al., 2010). $\mathrm{E}_{\mathrm{rs}}$ was lower with $\mathrm{PEEP}=8 \mathrm{cmH}_{2} \mathrm{O}$ than with PEEP $=3 \mathrm{cmH}_{2} \mathrm{O}$ during the ventilation protocol (Figure 3), likely suggesting some degree of recruitment, as depicted in Figure 1C. This is in line with a study on patients with acute respiratory distress syndrome that found a link between the $\mathrm{E}_{\mathrm{W}}$ increase caused by prone position and the improvement in oxygenation, possibly caused by lung recruitment (Pelosi et al., 1998).

\section{CT Scan Images}

A decrease in TLV was observed by CT scan in PPT group and even further in PPT-RC rats compared to CTRL animals (Figure 4). Increasing PEEP from 3 to $8 \mathrm{cmH}_{2} \mathrm{O}$ led to a significant increase in TLV only in CTRL group. In CTRL group, 
a high amount of lung units were normally aerated at PEEP $=3$ $\mathrm{cmH}_{2} \mathrm{O}$ and a larger PEEP increased aeration. During tidal breathing, CT disclosed a leftward shift of the histogram, suggesting ongoing hyperinflation (Figure 5A). This finding reinforces a previously reported association between $\mathrm{E}_{\mathrm{rs}}$ and imaging evidences of hyperinflation (Carvalho et al., 2006, 2008; Suarez-Sipmann et al., 2007).

In PPT and PPT-RC groups, increasing in chest wall elastance resulted in an overall loss of aeration. In PPT group, increasing PEEP led to a higher contribution of the fraction volume of the apex to TLV (Figure 6D), in agreement with the hypothesis that a normal rib cage elastance could allow lung inflation in the regions far from the diaphragm (Regli et al., 2012). Accordingly, with the rib cage restricted (PPT-RC group), an increase in PEEP from 3 to $8 \mathrm{cmH}_{2} \mathrm{O}$ augmented the contribution of air volume relative to TLV in the middle zones (ventro-dorsal and cephalo-caudal). Using this level of PEEP in animals with normal volume status and cardiac function, we think that this result should not be attributed to a change in intrathoracic and pulmonary blood volume (Luecke et al., 2004; Luecke and Pelosi, 2005). Moreover, the middle ventro-dorsal zone increased its tissue and gas contribution to TLV and the middle cephalocaudal zone tended to follow the same pattern $(p=0.053)$, suggesting lung recruitment closer to the diaphragm. As aforementioned, a PEEP level above $8 \mathrm{cmH}_{2} \mathrm{O}$ could have further recruited the lung and improved TLV (Loring et al., 2010).

\section{Limitations}

Our study has some limitations. Firstly, the direct clinical application of our findings should be carefully considered owing to differences between rat and human respiratory systems, such as the higher heterogeneity of lung expansion of the latter. Secondly, our protocol design did not include a higher PEEP, which could have led to larger lung recruitment in PPT-RC group. Thirdly, technical issues (esophageal catheter displacement and repositioning due to PEEP) prevented us to measure esophageal pressure and we were unable to evaluate the independent contributions of $\mathrm{E}_{\mathrm{L}}$ and $\mathrm{E}_{\mathrm{w}}$ to $\mathrm{E}_{\mathrm{rs}}$. Fourthly, in a clinical perspective, without an estimation of pleural pressure or an assessment of cardiac function and hemodynamics, we cannot conclude about heart-lung interactions. In the same line, we did not evaluate oxygenation, which could be affected by ventilation/perfusion mismatch resulting from changes in distribution of both ventilation (regional lung mechanics) and perfusion (possible redistribution of blood volume between body compartments and within the lungs). As a last note, our normal-lung rats do not represent patients with respiratory disease, which can further increase lung mechanics heterogeneity.

\section{REFERENCES}

Andersson, L. E., Bååth, M., Thörne, A., Aspelin, P., and Odeberg-Wernerman, S. (2005). Effect of carbon dioxide pneumoperitoneum on development of atelectasis during anesthesia, examined by spiral computed tomography. Anesthesiology 102, 293-299. doi: 10.1097/00000542-200502000-00009

\section{Clinical Applications}

Observing the mechanical aspects of the interaction of pneumoperitoneum, ribcage elastance and PEEP, the present study confirms our hypothesis that during pneumoperitoneum PEEP alveolar recruitment potential depends on rib cage elastance. We could speculate that, excluding clinical contraindications, patients with a stiffer or heavy ribcage would benefit from the application of high levels of PEEP, while individuals with a normal chest wall compliance would not, or, worse, their lungs could become even hyperinflated. Consequently, the same PEEP value cannot be used in all subjects.

\section{CONCLUSION}

Rib cage elastance might determine PEEP recruitment potential in the face of abdominal hypertension.

\section{AUTHOR CONTRIBUTIONS}

LC, MdA, AC, and WZ substantial contributions to the conception or design of the work. LC, MdA, GM-R, RO, TB, NC, $\mathrm{AC}$, and WZ substantial contributions to the acquisition, analysis, or interpretation of data for the work. LC, MdA, GM-R, NC, $\mathrm{UL}, \mathrm{AC}$, and WZ drafting the work or revising it critically for important intellectual content. LC, MdA, GM-R, RO, TB, NC, $\mathrm{UL}, \mathrm{AC}$, and $\mathrm{WZ}$ final approval of the version to be published. LC, MdA, GM-R, RO, TB, NC, UL, AC, and WZ agreement to be accountable for all aspects of the work in ensuring that questions related to the accuracy or integrity of any part of the work are appropriately investigated and resolved.

\section{FUNDING}

This study was supported by The Brazilian Council for Scientific and Technological Development (CNPq) (Grant Nos. 470495/2012-0, 300531/2012-5, and 400941/2012-0), and The Carlos Chagas Filho Rio de Janeiro State Research Supporting Foundation (FAPERJ) (Grant Nos. E-26/110.781/2010, E-26/110.385/2010, E-26/103.184/2011, E-26/201.450/2014, and E-26/203.344/2015).

\section{ACKNOWLEDGMENTS}

We would like to thank Mrs. Aline Santos e Silva, Mr. Antonio Carlos de Souza Quaresma, and Mr. Diego Vinicius da Silva Ribeiro for their skillful technical assistance.

Camilo, L. M., Ávila, M. B., Cruz, L. F. S., Ribeiro, G. C. M., Spieth, P. M., Reske, A. A., et al. (2014). Positive end-expiratory pressure and variable ventilation in lung-healthy rats under general anesthesia. PLoS One 9:e110817. doi: 10.1371/ journal.pone.0110817

Carvalho, A. R., Bergamini, B. C., Carvalho, N. S., Cagido, V. R., Neto, A. C., Jandre, F. C., et al. (2013). Volume-independent elastance: a useful parameter 
for open-lung positive end-expiratory pressure adjustment. Anesth. Analg. 116, 627-633. doi: 10.1213/ANE.0b013e31824a95ca

Carvalho, A. R., Spieth, P. M., Pelosi, P., Vidal Melo, M. F., Koch, T., Jandre, F. C., et al. (2008). Ability of dynamic airway pressure curve profile and elastance for positive end-expiratory pressure titration. Intensive Care Med. 34, 2291-2299. doi: 10.1007/s00134-008-1301-7

Carvalho, A. R. S., Jandre, F. C., Pino, A. V., Bozza, F. A., Salluh, J. I., Rodrigues, R., et al. (2006). Effects of descending positive end-expiratory pressure on lung mechanics and aeration in healthy anaesthetized piglets. Crit. Care 10:R122.

Cinnella, G., Grasso, S., Spadaro, S., Rauseo, M., Mirabella, L., Salatto, P., et al. (2013). Effects of recruitment maneuver and positive end-expiratory pressure on respiratory mechanics and transpulmonary pressure during laparoscopic surgery. Anesthesiology 118, 114-122. doi: 10.1097/ALN.0b013e3182746a10

Cortes-Puentes, G. A., Gard, K. E., Adams, A. B., Faltesek, K. A., Anderson, C. P., Dries, D. J., et al. (2013). Value and limitations of transpulmonary pressure calculations during intra-abdominal hypertension. Crit Care Med. 41, 1870-1877. doi: 10.1097/CCM.0b013e31828a3bea

Cortes-Puentes, G. A., Keenan, J. C., Adams, A. B., Parker, E. D., Dries, D. J., and Marini, J. J. (2015). Impact of chest wall modifications and lung injury on the correspondence between airway and transpulmonary driving pressures. Crit Care Med. 43, e287-e295. doi: 10.1097/CCM.0000000000001036

Fahy, B. G., Barnas, G. M., Flowers, J. L., Nagle, S. E., and Njoku, M. J. (1995). The effects of increased abdominal pressure on lung and chest wall mechanics during laparoscopic surgery. Anesth. Analg. 81, 744-750.

Formenti, P., Graf, J., Cortes, G. A., Faltesek, K., Gard, K., Adams, A. B., et al. (2012). Experimental intra-abdominal hypertension attenuates the benefit of positive end-expiratory pressure in ventilating effusion-compressed lungs* Crit. Care Med. 40, 2176-2181. doi: 10.1097/CCM.0b013e318250aa40

Futier, E., Constantin, J.-M., Pelosi, P., Chanques, G., Kwiatkoskwi, F., Jaber, S., et al. (2010). Intraoperative recruitment maneuver reverses detrimental pneumoperitoneum-induced respiratory effects in healthy weight and obese patients undergoing laparoscopy. Anesthesiology 113, 1310-1319. doi: 10.1097/ ALN.0b013e3181fc640a

Gattinoni, L., Pesenti, A., Avalli, L., Rossi, F., and Bombino, M. (1987). Pressurevolume curve of total respiratory system in acute respiratory failure. Computed tomographic scan study. Am. Rev. Respir. Dis. 136, 730-736. doi: 10.1164/ ajrccm/136.3.730

Loring, S. H., Behazin, N., Novero, A., Novack, V., Jones, S. B., O’Donnell, C. R., et al. (2014). Respiratory mechanical effects of surgical pneumoperitoneum in humans. J. Appl. Physiol. 117, 1074-1079. doi: 10.1152/japplphysiol.00552.2014

Loring, S. H., Pecchiari, M., Della Valle, P., Monaco, A., Gentile, G., and D'Angelo, E. (2010). Maintaining end-expiratory transpulmonary pressure prevents worsening of ventilator-induced lung injury caused by chest wall constriction in surfactant-depleted rats. Crit. Care Med. 38, 2358-2364. doi: 10.1097/CCM.0b013e3181fa02b8

Lowhagen, K., Lundin, S., and Stenqvist, O. (2010). Regional intratidal gas distribution in acute lung injury and acute respiratory distress syndrome assessed by electric impedance tomography. Minerva Anestesiol. 76, 1024-1035.

Luecke, T., and Pelosi, P. (2005). Clinical review: positive end-expiratory pressure and cardiac output. Crit. Care 9, 607-621. doi: 10.1186/cc3877

Luecke, T., Roth, H., Herrmann, P., Joachim, A., Weisser, G., Pelosi, P., et al. (2004). Assessment of cardiac preload and left ventricular function under increasing levels of positive end-expiratory pressure. Intensive Care Med. 30, 119-126. doi: 10.1007/s00134-003-1993-7
Maracajá-Neto, L. F., Verçosa, N., Roncally, A. C., Giannella, A., Bozza, F. A., and Lessa, M. A. (2009). Beneficial effects of high positive end-expiratory pressure in lung respiratory mechanics during laparoscopic surgery. Acta Anaesthesiol. Scand. 53, 210-217. doi: 10.1111/j.1399-6576.2008.01826.x

Martin-Lefèvre, L., Ricard, J.-D., Roupie, E., Dreyfuss, D., and Saumon, G. (2001). Significance of the changes in the respiratory system pressure-volume curve during acute lung injury in rats. Am. J. Respir. Crit. Care Med. 164, 627-632. doi: 10.1164/ajrccm.164.4.2008018

Moreira, L. F., Gobbi, C. F., Feijóo, M., Rocco, P. R., and Zin, W. A. (1997). Respiratory mechanics and morphometric changes during pneumoperitoneum in normal rats. Eur. Respir. J. 10, 1321-1326. doi: 10.1183/09031936.97. 10061321

Mutoh, T., Lamm, W. J., Embree, L. J., Hildebrandt, J., and Albert, R. K. (1991). Abdominal distension alters regional pleural pressures and chest wall mechanics in pigs in vivo. J. Appl. Physiol. 70, 2611-2618. doi: 10.1152/jappl. 1991.70.6.2611

Pelosi, P., Tubiolo, D., Mascheroni, D., Vicardi, P., Crotti, S., Valenza, F., et al. (1998). Effects of the prone position on respiratory mechanics and gas exchange during acute lung injury. Am. J. Respir. Crit. Care Med. 157, 387-393. doi: 10.1164/ajrccm.157.2.97-04023

Regli, A., Chakera, J., De Keulenaer, B. L., Roberts, B., Noffsinger, B., Singh, B., et al. (2012). Matching positive end-expiratory pressure to intra-abdominal pressure prevents end-expiratory lung volume decline in a pig model of intraabdominal hypertension*. Crit. Care Med. 40, 1879-1886. doi: 10.1097/CCM. ob013e31824e0e80

Runck, H., Schumann, S., Tacke, S., Haberstroh, J., and Guttmann, J. (2012). Effects of intra-abdominal pressure on respiratory system mechanics in mechanically ventilated rats. Respir. Physiol. Neurobiol. 180, 204-210. doi: 10.1016/j.resp. 2011.11.007

Suarez-Sipmann, F., Böhm, S. H., Tusman, G., Pesch, T., Thamm, O., Reissmann, H., et al. (2007). Use of dynamic compliance for open lung positive end-expiratory pressure titration in an experimental study. Crit. Care Med. 35, 214-221. doi: 10.1097/01.CCM.0000251131.40301.E2

Valenza, F., Chevallard, G., Fossali, T., Salice, V., Pizzocri, M., and Gattinoni, L. (2010). Management of mechanical ventilation during laparoscopic surgery. Best Pract. Res. Clin. Anaesthesiol. 24, 227-241.

Valenza, F., Chevallard, G., Porro, G. A., and Gattinoni, L. (2007a). Static and dynamic components of esophageal and central venous pressure during intraabdominal hypertension. Crit. Care Med. 35, 1575-1581.

Valenza, F., Vagginelli, F., Tiby, A., Francesconi, S., Ronzoni, G., Guglielmi, M., et al. (2007b). Effects of the beach chair position, positive end-expiratory pressure, and pneumoperitoneum on respiratory function in morbidly obese patients during anesthesia and paralysis. Anesthesiology 107, 725-732.

Conflict of Interest Statement: The authors declare that the research was conducted in the absence of any commercial or financial relationships that could be construed as a potential conflict of interest.

Copyright (c) 2018 Comuzzi, de Abreu, Motta-Ribeiro, Okuro, Barboza, Carvalho, Lucangelo, Carvalho and Zin. This is an open-access article distributed under the terms of the Creative Commons Attribution License (CC BY). The use, distribution or reproduction in other forums is permitted, provided the original author(s) and the copyright owner(s) are credited and that the original publication in this journal is cited, in accordance with accepted academic practice. No use, distribution or reproduction is permitted which does not comply with these terms. 